$\xi=1$ 国

\title{
Effects of pre-pregnancy maternal body mass index on gestational diabetes mellitus
}

\author{
Priya Shirley Muller ${ }^{1 *}$, M. Nirmala ${ }^{2}$ \\ ${ }^{1}$ Research Scholar, Department of Mathematics, Sathyabama University, Chennai \\ ${ }^{2}$ Professor, Department of Mathematics, Sathyabama University, Chennai \\ *Corresponding author E-mail: priyamuller@gmail.com
}

\begin{abstract}
The prevalence of both obesity and Gestational Diabetes Mellitus (GDM) is increasing worldwide. Overweight and obesity are abnormal or excessive fat accumulation that presents a risk to health. The presence of obesity has, in particular, a significant impact on both maternal and fetal complications associated with GDM. These complications can be addressed, at least in part, by good glycaemic control during pregnancy. The objective of the study is to classify GDM and non-GDM patients based on pre-pregnancy maternal Body Mass Index (BMI) and to assess and quantify the risk for GDM according to BMI.
\end{abstract}

Keywords: Gestational Diabetes Mellitus; Body Mass Index; Obesity; Discriminant Analysis; Risk Factor.

\section{Introduction}

\subsection{Gestational diabetes mellitus}

GDM is defined as any degree of impaired glucose tolerance during gestation and affects about $3 \%$ to $21 \%$ of all pregnancies. Nearly all women (90\%) with GDM are normoglycaemic after delivery. However, they are at risk for recurrent GDM, impaired glucose tolerance and overt diabetes in future. One-third to twothirds of women with GDM will have GDM in a subsequent pregnancy. Women who have a recurrence tend to be older, have higher parity and have a greater increase in weight between their pregnancies than women without a recurrence. Higher infant birth weight in the index pregnancy and higher maternal pre-pregnancy weight have also been associated with recurrent GDM [6]. As regards long-term risks, as many as $20 \%$ of women with GDM have impaired glucose tolerance during the early postpartum period. The cumulative incidence of future diabetes ranges from $2.6 \%$ to $70 \%$, with the greatest increase in risk in the first five years after a pregnancy with GDM and a plateau in risk after ten years [5].

GDM and type 2 diabetes share many common risk factors including overweight and obesity and GDM is considered by many to be a precursor of type 2 diabetes. Waist circumference and BMI are the strongest anthropometric measures associated with development of type 2 diabetes in women with GDM. Type 2 diabetes develops in $50-75 \%$ of obese women with a history of GDM [3]. In addition to the increased risk of obese women developing GDM, there is also an increased risk of type 2 diabetes, characterized by whole-body insulin resistance and higher plasma insulin concentrations. On the other hand, inter-pregnancy weight gain and increasing maternal age worsens the risk of developing GDM. The rate of fetal or neonatal death in the offspring of women with type2 diabetes or GDM is higher than in non-diabetic controls; this mainly occurs as late fetal death. There is also a strong relationship between the perinatal mortality rate and maternal obesity in pregnant women with type2 diabetes. Major complications of labour in obese women include labour induction failure, failure to progress in the first stage of labour, meconium-stained amniotic fluid, malpresentation and shoulder dystocia.

\subsection{Obesity}

The Body Mass Index or Quetelet index is defined as the body mass divided by the square of the body height. It is expressed in units of $\mathrm{kg} / \mathrm{m}^{2}$ universally, resulting from mass in kilograms and height in meters. The World Health Organization (WHO) and the National Institutes of Health define: underweight as a body mass index of less than 18.5 , normal weight as a BMI of 18.5-24.9, overweight as a BMI of 25-29.9, and obesity as a BMI of 30 and above. Obesity is further characterized by BMI into class I (30-34.9), class II (35-39.9), and class III (>40).

Overweight and obesity are defined as excessive or abnormal fat accumulation that would most possibly impair health. As a rule, women have more body fat than men and it is widely agreed that men with $>30 \%$ body fat and women with $>25 \%$ body fat are obese. Obesity has now become an epidemic. The WHO estimated in the year 2000 that as many as 300 million people worldwide were clinically obese. Obesity is associated with infertility and with many pregnancy complications. Moreover, it is associated with GDM, which increases the risk of these complications. As the prevalence of obesity is increasing, so is the number of women in the reproductive age who are overweight and obese. The average BMI is increasing among all age categories and women enter pregnancy at higher weights. Women are also more likely to retain gestational weight with each pregnancy.

\section{Literature survey}

Entering pregnancy with overweight, obesity or gaining excessive gestational weight could increase the risk of GDM, which is associated with negative consequences for both the mother and the 
offspring. Studies published between January 1975 and January 2015 on the relationship between GDM, pre-pregnancy BMI, gestational weight gain and nutritional prevention strategies suggest that maternal obesity assessed by pre-pregnancy BMI is associated with an increased risk of GDM. They also show an association between gestational weight gain and increased risk for GDM. Higher dietary fat and lower carbohydrate intakes during pregnancy appear to be associated with a higher risk for GDM, independent of pre-pregnancy BMI.

High maternal BMIs have been consistently associated with an increased risk of GDM in the literature. In a meta-analysis estimating the magnitude of GDM risk among women with high prepregnancy BMIs, Chu et al. found that GDM risk increases substantially with increasing pre-pregnancy BMI [2]. Moreover, a dose-response relationship between increasing BMI and type 2 diabetes has been described in the general population, even within the normal BMI category [10]. Several randomized trials have demonstrated that weight loss and increased physical activity reduce the risk of type 2 diabetes in individuals at high risk, including women with a history of GDM [8]. Similarly, evidence suggests that GDM risk is reduced in women who engage in high levels of physical activity [11]. Therefore, to the extent that prepregnancy overweight and obesity cause GDM, reducing prepregnancy weight in these women should reduce diabetes related adverse pregnancy outcomes. Weight loss and healthy lifestyle can help to prevent type 2 diabetes and also appear to reduce the risk of GDM. Sustaining this weight loss beyond pregnancy should reduce women's future risk for type 2 diabetes [1].

Although GDM risk increases substantially with increasing prepregnancy body mass index, the percentage of GDM specifically attributable to overweight and obesity is unknown. The objective of this article was to review scientific evidence regarding the association between obesity and GDM and to determine the percentage of GDM potentially attributable to overweight and obesity.

\section{Methodology}

Discriminant Analysis has been applied in various domains in medical diagnosis. It is a commonly accepted statistical tool, which can generate excellent models. Since discriminant analysis is easily used and analyzed and provides coefficients such as probability ratio to express each independent variable's impact on the model, it is frequently applied in biomedicine models. In this study, DA is used to identify the most significant factors of GDM. The goal of discriminant analysis is to predict group membership from a set of predictors and logistic discrimination allows predicting a discrete outcome such as group membership from a set of variables that may be continuous, discrete and dichotomous [4]. Discriminant analysis is a multivariable technique that separates distinct sets of observations and attributes new observations to predefined sets. Statistical problem is to develop a law (diagnosis or classification function) on the basis of population size. According to this law new samples with no clear attribution are attributed to one the populations. Fisher discriminant analysis can be pointed out as one of the most known functions applied in discriminant analysis.

The discriminant function score can be generated with unstandardized discriminant function scores and raw scores. To maximize the differences between the two groups, the discriminant function coefficients are chosen. The mean over all the discriminant function coefficients is zero while the standard deviation is equal to one. The mean discriminant function coefficient is calculated for each group which is called a centroid. This is created in the reduced space created by the discriminant function reduced from the initial predictor variables. The dimensions along which the groups differ can be shown by the differences in the location of these centroids. The groups are differentiated once the discriminant functions are determined. The utility of these functions can be examined through their ability to correctly classify each data point to their a priori groups. Classification functions are acquired from the linear discriminant functions to achieve this purpose. Different classification functions are used that are best suited for equal or unequal samples in each group.

For cases with an equal sample size for each group the classification function coefficient $\mathrm{C}_{\mathrm{j}}$ is as follows:

$$
C_{j}=c_{j 0}+c_{j 1} x_{1}+c_{j 2} x_{2}+\ldots+c_{j p} x_{p}
$$

For the $\mathrm{j}^{\text {th }}$ group, $\mathrm{j}=1 \ldots \mathrm{k}, \mathrm{x}=$ raw scores of each predictor, $\mathrm{c}_{\mathrm{j} 0}=\mathrm{a}$ constant. If $\mathrm{M}=$ column matrix of means for group $\mathrm{j}$ and $\mathrm{W}=$ within-group variance-covariance matirix, then the constant $c_{j 0}=(-$ 1/2) $\mathrm{C}_{\mathrm{j}} \mathrm{M}_{\mathrm{j}}$

For unequal sample size in each group,

$$
C_{j}=c_{j 0}+\sum_{i=1}^{p} c_{i j} x_{i}+\ln \left(\frac{n_{j}}{N}\right)
$$

$\mathrm{n}_{\mathrm{j}}=$ size in group $\mathrm{j}, \mathrm{N}=$ total sample size .

Discriminant function analysis is the reverse process of Multivariate Analysis of Variance (MANOVA). The dependent variables are the predictors and the independent variables are the groups in MANOVA whereas in discriminant analysis, the dependent variables are the groups and the independent variables are the predictors. Several variables are generally included in a study to see which ones contribute to the discrimination between groups. The process of testing significance of a set of discriminant functions in discriminant function analysis is computationally identical to MANOVA. There is a matrix of total variances and covariances; similarly there is a matrix of pooled within-group variances and covariances. In order to determine whether there are any significant differences with regard to all variables between groups, the two matrices are compared using multivariate $\mathrm{F}$ tests. The multivariate test is first performed and if statistically found significant, one proceeds to see which of the variables have significantly different means across the groups.

\section{Data collection}

The real time data was collected from past patient records in a multi-specialty hospital in Chennai, Tamil Nadu, India. The patient data sets of 332 records of which 188 were of multi gravida patients, each consisting of ten parameters, was extracted from the outgoing patient's records during the period January to May, 2013. On consultation with gynaecologists and taking into account the several factors that are clinically relevant for a pregnant woman to develop GDM, the study variables were chosen.

\begin{tabular}{|c|c|c|}
\hline $\begin{array}{l}\mathrm{S} \\
\text { No }\end{array}$ & Study Variable & $\begin{array}{l}\text { Classification Network Varia- } \\
\text { ble type }\end{array}$ \\
\hline 1 & Age & Integer [continuous] \\
\hline 2 & Family history of diabetes & $\mathrm{Y}$ or $\mathrm{N}$ [character] \\
\hline 3 & Pre pregnancy body mass index & Integer [continuous] \\
\hline 4 & History of GDM & $\mathrm{Y}$ or $\mathrm{N}$ [character] \\
\hline 5 & $\begin{array}{l}\text { Delivery of a large infant } \\
(>3.8 \mathrm{Kg})\end{array}$ & $\mathrm{Y}$ or $\mathrm{N}$ [character] \\
\hline 6 & History of miscarriage & $\mathrm{Y}$ or $\mathrm{N}$ [character] \\
\hline 7 & $\begin{array}{l}\text { Abnormal baby in previous } \\
\text { pregnancy }\end{array}$ & $\mathrm{Y}$ or $\mathrm{N}$ [character] \\
\hline 8 & History of stillbirth & $\mathrm{Y}$ or $\mathrm{N}$ [character] \\
\hline 9 & History of Infections & $\mathrm{Y}$ or $\mathrm{N}$ [character] \\
\hline 10 & $\begin{array}{l}\text { History of Polycystic ovary } \\
\text { syndrome }\end{array}$ & $\mathrm{Y}$ or $\mathrm{N}$ [character] \\
\hline
\end{tabular}

Table 1: The Parameters Chosen for the Study

Table 1 shows the variables chosen which are clinically relevant for the study. The first three variables used in the model involve general information like age, family history of diabetes in first degree relatives and Body Mass Index. Fourth to eighth variables deal with previous pregnancy information such as presence of 
GDM, birth of a baby which weighed more than $3.8 \mathrm{Kg}$, death of a baby before 20 weeks, birth of a baby with defects in spinal cord, heart or brain, death of a baby after 20 weeks respectively. The last two reveal information on history of urinary, skin or vaginal infections and presence of polycystic ovary syndrome [7]. Eight of the ten variables used are binary variables, where 0 indicates nonoccurrence and 1 indicates occurrence.

\section{Results and discussion}

Risk factors of diabetes have been long under investigation and analysis has been going on in various studies and communities. However, there is no considerable number of studies to examine the risk factors of GDM on pregnant women. Identification of these risk factors demands accurate and sensitive statistical tools. To estimate the most influential risk factors of GDM, discriminant analysis was performed on the entire sample. Results were analyzed using the Statistical Package for Social Sciences (SPSS) for Windows version 20.0.

Table 2: Tests of Equality of Group Means in the Discriminant Analysis Model

\begin{tabular}{llll} 
Model & & & \\
\hline Variables & $\begin{array}{l}\text { Wilks' } \\
\text { Lambda }\end{array}$ & $\begin{array}{l}\text { P Val- } \\
\text { ue }\end{array}$ & F Value \\
\hline Age & 0.970 & 5.850 & $0.017^{*}$ \\
Family history of diabetes & 0.871 & 27.594 & $<0.001^{* *}$ \\
Pre pregnancy body mass index & 0.920 & 16.130 & $<0.001^{* *}$ \\
History of GDM & 0.660 & 95.894 & $<0.001^{* *}$ \\
Delivery of a large infant & 0.965 & 6.657 & $0.011^{*}$ \\
History of miscarriage & 0.962 & 7.283 & $0.008^{* *}$ \\
Abnormal baby in previous & 0.984 & 2.953 & 0.087 \\
pregnancy & 0.984 & 3.030 & 0.083 \\
History of stillbirth & 0.966 & 6.455 & $0.012^{*}$ \\
Infections (Urinary, Skin, Vagi- & & & \\
nal) & 0.988 & 2.190 & 0.141 \\
History of Polycystic ovary & & & \\
syndrome & & &
\end{tabular}

Note: $* *$ denotes significant at $1 \%$ level

$*$ denotes significant at $5 \%$ level

ANOVA F test of mean differences in DA uses Wilks' lambda, in which the smaller the lambda value for an independent variable, the more that variable contributes to the discriminant function [9] Lambda value varies from 0 to 1 , wherein 0 indicates that the group means differ (thus the more the variable differentiates the groups) and a value of 1 indicates that all group means are the same. Thus the F test of Wilks' lambda shows which variables contributions are significant. The correlations of each variable with each discriminant function are shown by the structure matrix table in SPSS. These simple Pearsonian correlations are called discriminant loadings or correlations or structure coefficients. There will be more than one discriminant function when the dependent has more than two categories. One can gain insight into how to name each function by identifying the largest absolute correlations associated with each discriminant function. Wilks' Lambda test with $\mathrm{p}<0.001$ indicates discriminant analysis significance.

The results from table 2 indicated that of all the variables, prepregnancy BMI, history of GDM, and family history of diabetes had the smallest $\mathrm{p}$-values and hence they were most significantly associated with occurrence of GDM during current pregnancy. Further, history of miscarriage had small $\mathrm{p}$ value ranging from 0.000 to 0.010 and hence was significant at $1 \%$ level. The variables age, delivery of large infant and history of infections had $\mathrm{p}$ values lying in the range 0.011 to 0.050 and consequently were significant at $5 \%$ level.

Population-based risk estimates are needed to calculate the percentage of GDM cases that could potentially be prevented if all women who are overweight or obese had a GDM risk equivalent to that of women of normal weight. The percentage of GDM attributable to overweight and obesity was calculated as a means of better understanding the potential effects of weight management on GDM prevalence.

\begin{tabular}{llll}
\multicolumn{3}{c}{ Table 3: BMI Based Classification of GDM } \\
\hline BMI Catego- & Total No of & No of GDM & No of non-GDM \\
ry & Patients & Patients & Patients \\
\hline Underweight & 04 & 00 & 04 \\
Normal & 101 & 25 & 76 \\
Overweight & 74 & 33 & 41 \\
$\begin{array}{l}\text { Obese(Class } \\
\text { 1) }\end{array}$ & 08 & 05 & 03 \\
$\begin{array}{l}\text { Obese(Class } \\
\text { 2) }\end{array}$ & 01 & 01 & 00 \\
\hline
\end{tabular}

Table 3 shows classification of GDM and non-GDM patients based on BMI. In the underweight $(<18.5)$ category, no pregnant woman had GDM. In the normal weight (18.5-24.9) category, 25 out of 101 women had GDM, which means $24.75 \%$ of the normal weight population had GDM. There were 74 pregnant women in the overweight (25-29.9) category, of which 33 had GDM, implying an astounding $44.59 \%$ of the overweight pregnant women had GDM. In the obese class 1 (30-34.9) category, 5 out of 8 women namely $62.5 \%$ of the pregnant women had GDM. Lastly, the only pregnant woman who belonged to the obese class 2 (35-39.9) category had GDM. Of the total 188 records, 64 pregnant women had GDM of which 33 of them belonged to the overweight BMI category. In other words, $51.56 \%$ of the GDM patients were overweight. Moreover, 39 out of the 64 GDM patients belonged to the overweight/obese category, which means a staggering $60.94 \%$ of the GDM patients were either overweight or obese. In other words, more than $60 \%$ of all women with GDM had a BMI of 25 or higher, whereas the remaining had a normal BMI

\section{Conclusion}

Using discriminant analysis model, it was found that prepregnancy BMI is the most significant factor risk factor of GDM. Moreover, an alarming $60.94 \%$ of GDM cases were found to be attributable to overweight and obesity alone. This article clearly proves that GDM risk increases substantially with increasing maternal BMI. The increasing prevalence of overweight and obesity and related conditions such as GDM and type 2 diabetes are already changing predictions of the cost of medical care in the future. Preventing GDM depends on preventing obesity in young women; preventing type 2 diabetes in obese women who have GDM depends on effective nutrition and physical activity interventions.

The above analysis can help public health officials estimate the potential effects of prevention interventions on GDM prevalence rates. Lifestyle interventions designed to reduce BMIs have the potential to lower GDM risk. Therefore, public health efforts to promote recommended levels of physical activity and healthy eating habits among women of reproductive age should be intensified. Increased physical activity in women who are sedentary and opting for healthy food choices rather than 'fast foods' may result in a better pregnancy outcome for both mother and child. Thus, weight management through nutritional prevention strategies could prove successful in reducing the risk for GDM.

\section{References}

[1] Artal R, Catanzaro RB, Gavard JA, Mostello DJ, Friganza JC., A lifestyle intervention of weight-gain restriction: diet and exercise in obese women with gestational diabetes mellitus, Appl Physiol Nutr Metab., 32(3):596-601, 2007. https://doi.org/10.1139/H07-024.

[2] Chu SY, Callaghan WM, Kim SY, et al., Maternal obesity and risk of gestational diabetes mellitus, Diabetes Care, 30(8):2070-2076, 2007. https://doi.org/10.2337/dc06-2559a.

[3] Gabbe S., Gestational diabetes mellitus, N Engl J Med., 315:10256, 1986. https://doi.org/10.1056/NEJM198610163151609.

[4] Hosmer D. W. and Lemeshow S. Applied Logistic Regression, John Wiley, New York, 1989 
[5] Kim C, Newton KM, Knopp RH., Gestational diabetes and the incidence of type 2 diabetes: a systematic review, Diabetes Care, 25:1862-8, 2002. https://doi.org/10.2337/diacare.25.10.1862.

[6] MacNeill S, Dodds L, Hamilton DC, et al. Rates and risk factors for recurrence of gestational diabetes, Diabetes Care, 24:659-62, 2001. https://doi.org/10.2337/diacare.24.4.659.

[7] Priya Shirley Muller, S.Meenakshi Sundaram, M.Nirmala, E.Nagarajan, Application of Computational Technique in Design of Classifier for Early Detection of Gestational Diabetes Mellitus, Applied Mathematical Sciences, 9 (67), pp 3327 - 3336, 2015. https://doi.org/10.12988/ams.2015.54319.

[8] Ratner RE., Prevention of type 2 diabetes in women with previous gestational diabetes, Diabetes Care, 30(suppl 2):S242-S245, 2007. https://doi.org/10.2337/dc07-s223.

[9] Rezaei Mansour, Zandkarimi Eghbal and Hashemian Amirhossein, Comparison of Artificial Neural Network, Logistic Regression and Discriminant Analysis Efficiency in Determining Risk Factors of Type 2 Diabetes, World Applied Sciences Journal 23 (11), pp 1522 1529, 2013.

[10] Shai I, Jiang R, Manson JE, et al., Ethnicity, obesity, and risk of type 2 diabetes in women: a 20-year follow-up study, Diabetes Care, 29(7):1585-1590, 2006. https://doi.org/10.2337/dc06-0057.

[11] Zhang C, Solomon CG, Manson JE, Hu FB., A prospective study of pregravid physical activity and sedentary behaviors in relation to the risk for gestational diabetes mellitus, Arch Intern Med., 166(5):543-548, 2006. https://doi.org/10.1001/archinte.166.5.543. 